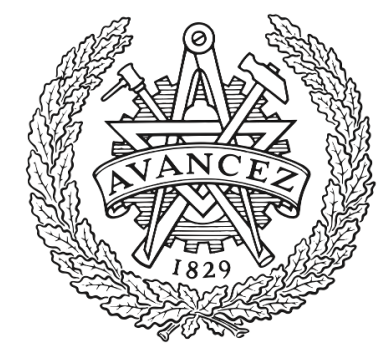

CHALMERS

UNIVERSITY OF TECHNOLOGY

\title{
A G-Band (140-220 GHz) planar stubbed branch-line balun in BCB technology
}

Downloaded from: https://research.chalmers.se, 2023-04-26 06:43 UTC

Citation for the original published paper (version of record):

Carpenter, S., Abbasi, M., Karandikar, Y. et al (2013). A G-Band (140-220 GHz) planar stubbed branch-line balun in BCB technology. 2013 3rd Asia-Pacific Microwave Conference, APMC 2013, Seoul, South Korea, 5-8 November 2013: 273-275. http://dx.doi.org/10.1109/APMC.2013.6695117

N.B. When citing this work, cite the original published paper. 


\title{
Applied Polymer
}

\section{Controlling Water Permeability of Composite Films of Polylactide Acid, Cellulose, and Xyloglucan}

\author{
Sofie Gårdebjer, ${ }^{1,2}$ Anette Larsson, ${ }^{1,2}$ Caroline Löfgren, ${ }^{3}$ Anna Ström ${ }^{1,2}$ \\ ${ }^{1}$ Department of Chemical and Biological Engineering, Chalmers University of Technology, 41296 Göteborg, Sweden \\ ${ }^{2}$ SuMo Biomaterials, VINN Excellence Center, Chalmers University of Technology, 41296 Göteborg, Sweden \\ ${ }^{3}$ Södra Skogsägarna ekonomisk förening, Södra Innovation, 43286 Väröbacka, Sweden \\ Correspondence to: A. Ström (E - mail: anna.strom@chalmers.se)
}

\begin{abstract}
To test the hypothesis that the introduction of a hydrophilic hemicellulose would affect viscoelastic properties and increase water permeability, xyloglucan (XG) was adsorbed onto the surface of microcrystalline cellulose (MCC) in water dispersion prior to the extrusion of 79-80 wt \% polylactide acid (PLA), 20 wt \% MCC, and 0-1 wt \% XG. For comparison, composites of PLA, MCC, and non-absorbed XG were produced. Analysis of thermal properties showed no differences for glass-transition or melting temperatures, but the crystallinity of the films increased with the addition of MCC and XG. Storage modulus of the composite materials increased with XG content; however, at higher humidities storage modulus decreased, probably because of lower interfacial adhesion. Water permeability through the films increased more with the addition of XG adsorbed to the MCC than with the MCC and XG simply mixed in the same amounts. () 2014 The Authors. Journal of Applied Polymer Science Published by Wiley Periodicals, Inc. J. Appl. Polym. Sci. 2015, 132, 41219.
\end{abstract}

KEYWORDS: cellulose and other wood products; composites; extrusion; films; viscosity and viscoelasticity

Received 21 March 2014; accepted 26 June 2014

DOI: 10.1002/app.41219

\section{INTRODUCTION}

Interest in plastics derived from alternative, non-petroleum sources has increased in recent years because they may reduce the environmental impact of plastics. ${ }^{1,2}$ Bioplastic materials can be made from plant, animal, or bacterial sources, and they can be biodegradable or non-biodegradable. As the cost of fossil fuels increase, bioplastics such as polylactide acid (PLA) become more competitive on the market. ${ }^{3}$ Bioplastics can be produced in common processing plants, enabling a relatively simple shift from petro-plastics to PLA-plastics. However, there are problems related to PLA, PLA is derived from food crops, and a major increase in production would negatively impact the world food supply. ${ }^{4}$ Furthermore, PLA has a low softening temperature (about $60^{\circ} \mathrm{C}$ ), which reduces the range of its application. ${ }^{5}$

To meet these challenges, different composites of PLA and cellulose have been produced. ${ }^{6-10}$ These composites have a better plastic storage modulus, which is an advantage both during their processing ${ }^{6}$ and in their use for food packaging (e.g., in drinking cups and blister packs). ${ }^{11}$ However, the increased strength in the plastic region gained from the use of a PLA/cellulose composite material is often accompanied by a decrease in the material's elongation, ${ }^{9}$ which is related to poor interfacial adhesion between the PLA and the cellulose. ${ }^{3,9,12}$ Attempts to improve adhesion between PLA and cellulose have included chemical surface treatments of the cellulose fibers ${ }^{13-15}$ or the addition of a compatibilizing agent. ${ }^{16,17}$ These procedures using organic solvents and pure cellulose are not always suitable for industrial processes, in which a simpler procedure and fewer steps to purify the cellulose are preferred. For example bleached Kraft pulp is a cellulosic material that incorporates both cellulose and hemicelluloses, its mixture with biodegradable material such as PLA, is currently of industrial interest. ${ }^{18}$ The properties of such composites are thus of interest, both from an academic and industrial point of view.

The gas and water permeability of PLA and its composites is interesting because the long-term goal is often to use these composites in cars, packaging, agricultural mulch films, or biomedical devices, in which a higher or lower permeability may be desired. ${ }^{11,19-21}$ Several studies have focused on the reduction of gas and water permeability in PLA and composites. ${ }^{22-24}$ Factors known to reduce the permeability of a polymeric material

This is an open access article under the terms of the Creative Commons Attribution-NonCommercial-NoDerivs License, which permits use and distribution in any medium, provided the original work is properly cited, the use is non-commercial and no modifications or adaptations are made.

(c) 2014 The Authors. Journal of Applied Polymer Science Published by Wiley Periodicals, Inc. 
are increased tortuosity path and increased crystallinity. ${ }^{24-26}$ Increasing the tortuosity path is obtained by adding an impermeable filler and increasing the crystallinity of PLA by annealing. Routes to increase water permeability have been rarely studied, ${ }^{27}$ despite their potential use in applications such as biomedical devices and agriculture. ${ }^{11,19-21}$ Incorporation of the hydrophilic hemicellulose xyloglucan $(\mathrm{XG})$, in which the water contact angle in films has been measured to $29^{\circ}$ (compared with $40^{\circ}$ for cellulose films), ${ }^{3}$ could be a potential way to control water permeability.

Xyloglucan is a thermostable hemicellulose extracted from nonfood sources, ${ }^{28}$ making it suitable for incorporation in bioplastic composites. XG has been shown to coat cellulose microfibrils, ${ }^{29}$ nanofibrils, ${ }^{30}$ and MCC. ${ }^{31,32}$ Additionally, a detailed study of atomic force measurements between PLA beads and cellulose films showed that the adhesion force was weak, but increased if the film was composed of pectin or XG instead of cellulose at a lower relative humidity $(\mathrm{RH} ; 2 \%){ }^{3}$ This was explained by the rise of capillary forces between pectin and/or XG films with PLA beads, because water molecules are present even at low $\mathrm{RH}$ in both pectin and XG films. In contrast, at a higher RH (56\%) the adhesion force was not affected by the film's composition (cellulose, XG, or pectin). The reduced capillary forces have also been observed and discussed in other studies. For example, Raj and co-workers measured the adhesion between PLA and cellulose by atomic force microscopy (AFM) and showed that the adhesion force was dominated by capillary forces up to $\sim 70 \% \mathrm{RH} .{ }^{33}$ Xiao and co-workers also measured the adhesion force between a hydrophilic $\mathrm{SiO}_{2}$ surface and a hydrophobic AFM tip of $\mathrm{Si}_{3} \mathrm{~N}_{4} \cdot{ }^{34}$ At an $\mathrm{RH}$ above $80 \%$ the adhesion force decreased drastically, also explained by reduced capillary forces at a higher $\mathrm{RH}$.

In the study reported here, we investigated the effect on water permeability of the addition of XG to a composite material consisting of PLA and MCC. We found that the water permeability of the composite films increases with the addition of XG, provided that the $\mathrm{XG}$ is adsorbed to the cellulose surface prior to the composite's production. In addition, the results of dynamic mechanical testing of the composite materials suggest that the adhesion between the filler (XG-covered MCC) and the matrix (PLA) depends on the RH, as has been suggested by others. ${ }^{3,33,34}$ This study clearly showed that the localization of $\mathrm{XG}$ is essential for the properties of the composite films; films with XG adsorbed to MCC have better adhesion and increased water permeability than films with the same composition but in which XG is not pre-adsorbed to the MCC.

\section{EXPERIMENTAL}

\section{Filler Preparation}

Xyloglucan from tamarind seed (Megazyme, Ireland) was added to water under stirring. The suspension was heated to $35^{\circ} \mathrm{C}$ and kept at that temperature overnight. MCC, Avicel PH101 (FMC Biopolymer, USA), was sieved to a particle size of 32 to $45 \mu \mathrm{m}$ (Retsch AS200, Germany). MCC was then dispersed in water and different amounts of the XG solution were added to obtain different XG : MCC ratios. The amounts of XG chosen to be pre-adsorbed to MCC were $0,4,10,15,25$, and $50 \mathrm{mg} \mathrm{XG/g}$ MCC. In addition, two dispersions containing $25 \mathrm{mg}$ XG were prepared to produce one material consisting only of PLA and $\mathrm{XG}$, and another material consisting of PLA, MCC, and XG, in which the surface of the MCC was not covered in advance. The XG : MCC ratios were chosen according to studies in which low XG : MCC ratios seemed to result in incomplete XG coverage of the MCC particles, whereas high XG : MCC ratios corresponded to saturated MCC particles. ${ }^{31,32}$ A total of eight different materials were studied, including the pure PLA material. The dispersions of MCC and XG were kept on magnetic stirring overnight at room temperature. The viscosity of the XG solution and the supernatants in the MCC $+\mathrm{XG}$ dispersions were determined using a Physica MCR300 Rheometer (Germany). The geometry used was a cone plate; the cone had a diameter of $50 \mathrm{~mm}$, an angle of $1^{\circ}$, and a truncation of $52 \mu \mathrm{m}$. The viscosities were determined over shear rates of $10-1000 \mathrm{~s}^{-1}$ at $20^{\circ} \mathrm{C}$. All dispersions were filtered using an Acrodisc glass-fiber filter with a pore size of $1 \mu \mathrm{m}$ purchased from Pall (USA). The MCC, XG, and the XG : MCC dispersions were freeze-dried at $-5^{\circ} \mathrm{C}$ overnight, using a FreeZone stoppering try dryer (Labconco, USA).

\section{Film Production}

Polylactide acid 3051D with a specific density of $1.25 \mathrm{~g} \mathrm{~cm}^{-3}$ and a molecular weight of $\sim 1.42 \times 10^{4} \mathrm{~g} \mathrm{~mol}^{-1}$ was purchased from Nature Works, USA. The freeze-dried MCC $+\mathrm{XG}$ powder was mixed with PLA at a mass ratio of $20: 80$, and the mixture was extruded in a twin-screw extruder at $170^{\circ} \mathrm{C}$ (Haake microcompounder, Rheomex CTW5, USA). To ensure good mixing of the melt, it was circulated for $5 \mathrm{~min}$ in the microcompounder. The cylindrical shaped extrudate was cut into pellets, which were pressed into films at $160^{\circ} \mathrm{C}$ for 5 min under a pressure of 5 bars. The pure PLA film and the PLA $+\mathrm{XG}$ and PLA $+\mathrm{MCC}+\mathrm{XG}$ films were produced in the same manner. The films were kept in a desiccator with silica orange at room temperature until further use.

\section{Film Morphology}

The microstructures of the films were analyzed using a scanning electron microscope (Leo Ultra 55 FEG-SEM, LeoElectron Microscopy, UK). Before the analysis, the films were drawn apart in an Instron device (Instron 5565A, USA) and the crosssections were surface-coated with a thin layer of gold to avoid charging the sample.

\section{Thermal Characteristics}

Differential scanning calorimetric measurements of the PLA and composite films were performed in a Perkin-Elmer Pyris 1, USA. Typically, samples of 4-6 mg of the films were placed in $50 \mu \mathrm{L}$ aluminum pans without holes. The program temperatures ranged from 10 to $180^{\circ} \mathrm{C}$, and the heating and cooling rate was set to $10^{\circ} \mathrm{C} \mathrm{min^{-1 }}$. $\mathrm{N}_{2}$ was used as the purging gas and empty pans were run in advance and used as a background. Samples were run in duplicates or larger sets. The crystallinity of the pure PLA films was calculated according to eq. (1):

$$
x_{c}=\frac{\Delta H_{m}-\Delta H_{\mathrm{cc}}}{\Delta H_{m}^{0}} \times 100 \%
$$

where $\Delta H_{m}^{0}$ is the heat of melting for $100 \%$ crystalline PLA, equal to $93.1 \mathrm{~J} \mathrm{~g}^{-135,36}$ and $\Delta H_{m}$ is the enthalpy of fusion during melting. $\Delta H_{\mathrm{cc}}$ represents the heat released during cold crystallization. $\Delta H_{m}$ and $\Delta H_{\mathrm{cc}}$ were determined from the peaks of 
the first heating curve. In the composite materials the crystallinity of the matrix polymer were calculated according to eq. $(2)^{37}$ :

$$
x_{c}=\frac{1}{1-w_{f}} \frac{\Delta H_{m}-\Delta H_{\mathrm{cc}}}{\Delta H_{m}^{0}} \times 100 \%
$$

where the weight fraction, $w_{f}$ of the matrix filler is subtracted.

\section{Viscoelastic Properties}

The viscoelastic properties of the films were tested in a dynamic mechanical analyzer (DMA Q-800, TA Instruments, USA). Film samples of $8.7 \mathrm{~mm}$ wide were mounted using a conventional film clamp. The average thicknesses of the films were $90 \mu \mathrm{m}$. The measurements were carried out at a frequency of $1 \mathrm{~Hz}$ and a strain of $0.05 \%$. Both values were verified to lie within the linear elastic region of the materials. The preload was set to $0.01 \mathrm{~N}$. An RH accessory (DMA-RH Accessory, TA instruments, USA) was connected to the instrument and a humidity sweep was performed with deionized water between $10 \%$ and $90 \% \mathrm{RH}$ at $25^{\circ} \mathrm{C}$. The $\mathrm{RH}$ was increased by $1 \%$ during 10 minutes after an equilibration period of $60 \mathrm{~min}$.

\section{Permeability Measurements}

Permeability measurements were performed in diffusion cells, as described by others. ${ }^{38,39}$ Briefly, the diffusion cells consist of a donor and an acceptor chamber, with a film separating the chambers. The thickness of the films was recorded three times before the permeability measurement. Nearly $15 \mathrm{~mL}$ of MilliQ-water was added to each chamber, $10 \mu \mathrm{L}$ of ${ }^{3}[\mathrm{H}]$-labeled water was added to the donor chamber, and the solution was stirred by placing the diffusion chambers on a rotating table at $50 \mathrm{rpm}$ (Edmund Bühler 7400 , Germany). Because of the large difference in radioactivity between the donor and acceptor chambers, the donor chamber concentration was constant during the entire experiment. Following the permeation measurement samples of $500 \mu \mathrm{L}$ were taken from the acceptor chamber and immediately replaced with an equal amount of pure MilliQ-water. Samples were assayed with a scintillation liquid, Ultima Gold, and run in a liquid scintillation analyzer (Tri-Carb B2810TR, Perkin-Elmer, USA). The mass transfer rate was calculated according to eq. (3), using Fick's first law for steady state mass transfer rate through a film:

$$
\frac{d m}{d t} A=\dot{m} A=\mathrm{DKA} \frac{c_{d}-c_{a}}{h}=\mathrm{PA} \frac{c_{d}-c_{a}}{h}
$$

where $c_{d}$ and $c_{a}$ correspond to the donor and acceptor chamber concentrations respectively, $A$ is the membrane area, $h$ is the film thickness, and $P$ is the permeability. The mass transfer rate, $\dot{m}$, was calculated from the linear slope in a plot of accumulated water against time. Permeability was multiplied by the average thickness of the films.

\section{RESULTS AND DISCUSSION}

\section{Material Characterization}

The MCC particles were covered with XG in a stirred water suspension overnight. Partial coverage of MCC was anticipated with the lower amounts of added XG (4 and $10 \mathrm{mg} \mathrm{g}^{-1} \mathrm{MCC}$ ) and complete coverage and/or excess XG was expected with 25 and $50 \mathrm{mg} \mathrm{g}^{-1}$ MCC. $^{31,32}$ On removal of the beakers from the stirrer, the appearance of the MCC $+\mathrm{XG}$ dispersions varied. The sedimentation rate was lower for the dispersions containing a higher XG : MCC ratio and the sedimentation height was higher. That this effect was not related to the increased viscosity of the bulk was verified by measuring the viscosity of the supernatant of the MCC and XG : MCC dispersions and the XG solutions. The results showed highest viscosity for the $\mathrm{XG}$ solution, followed by the MCC and finally the XG : MCC dispersions. The highest viscosity is obtained in the pure XG samples likely because the XG is fully dissolved and contributes fully to the viscosity of the solution. The absorption of XG onto the MCC will result in a reduced $\mathrm{XG}$ concentration in the solution and thus a reduced contribution to the viscosity. Because the XG are attached to the MCC particles, the tendency for the MCC to aggregate will be reduced, mainly because of the steric stabilization gained by the absorbed XG. Such reasoning is in line with common practice in reducing protein sedimentation and adsorbing polymers to enhance steric stabilisation of colloidal particles. ${ }^{40,41}$

After the extrusion and hot-melt pressing of the films, the film consisting of pure PLA was transparent. The addition of $25 \mathrm{mg}$ XG to PLA resulted in an inhomogeneous film with several clusters of XG observed, indicating poor dispersion of XG in the PLA matrix by the current process. The PLA films consisting of MCC that had been covered with XG showed a more homogeneous structure and a better dispersion of the MCC particles than the films to which MCC and XG were added separately.

Cross-sections of the films were studied using SEM after fracture. Figure 1(a) shows the sieved MCC on a flat surface without any further treatment. The particles are the desired sieved size of $32-45 \mu \mathrm{m}$; however, their shapes are irregular, going from cylindrical to spherical. SEM images in Figure 1(b-e) show the cross-section of the fracture of the films obtained upon tensile testing at room temperature. The cross-section of a pure PLA film appears smooth despite the tensile fracture [Figure 1(b)], while the interior of the film containing $20 \%$ MCC [Figure 1(c)] seems rough, with easily identified MCC particles interspersed within the PLA matrix. The cross-sections of the films containing 20\% MCC and 4 and $50 \mathrm{mg}$ XG/g MCC [Figure $1(\mathrm{~d}, \mathrm{e})$ ] appears similar to the cross-sections of PLA+MCC films without added XG. The interiors of the films containing the other additional amounts of pre-adsorbed XG were similar to those shown in Figure 1(b-e) and are therefore not shown.

\section{Thermal and Viscoelastic Characteristics of the Composite Films}

The results of the differential scanning calorimetric measurements (Table I) show that there are no major differences in either glass-transition or melting temperature for the different films, meaning that the addition of either MCC or XG contributes neither to plasticization or adherence to PLA. As shown in Table I the cold crystallization temperature decreased when MCC was added. Pure PLA crystallized at $120^{\circ} \mathrm{C}$, and the composite films (except the one in which PLA, MCC, and XG were mixed without pre-adsorption of the XG to MCC) crystallized around $100^{\circ} \mathrm{C}$. The lower cold crystallization temperature for the PLA+MCC composites indicates that MCC acts as a nucleating agent in the films. ${ }^{5}$ Table I also shows crystallinity of around $10 \%$ in the composite films and only $1 \%$ in the pure PLA film, which strengthen the idea of MCC acting as nucleating agents. All the composite films contained the same amount 


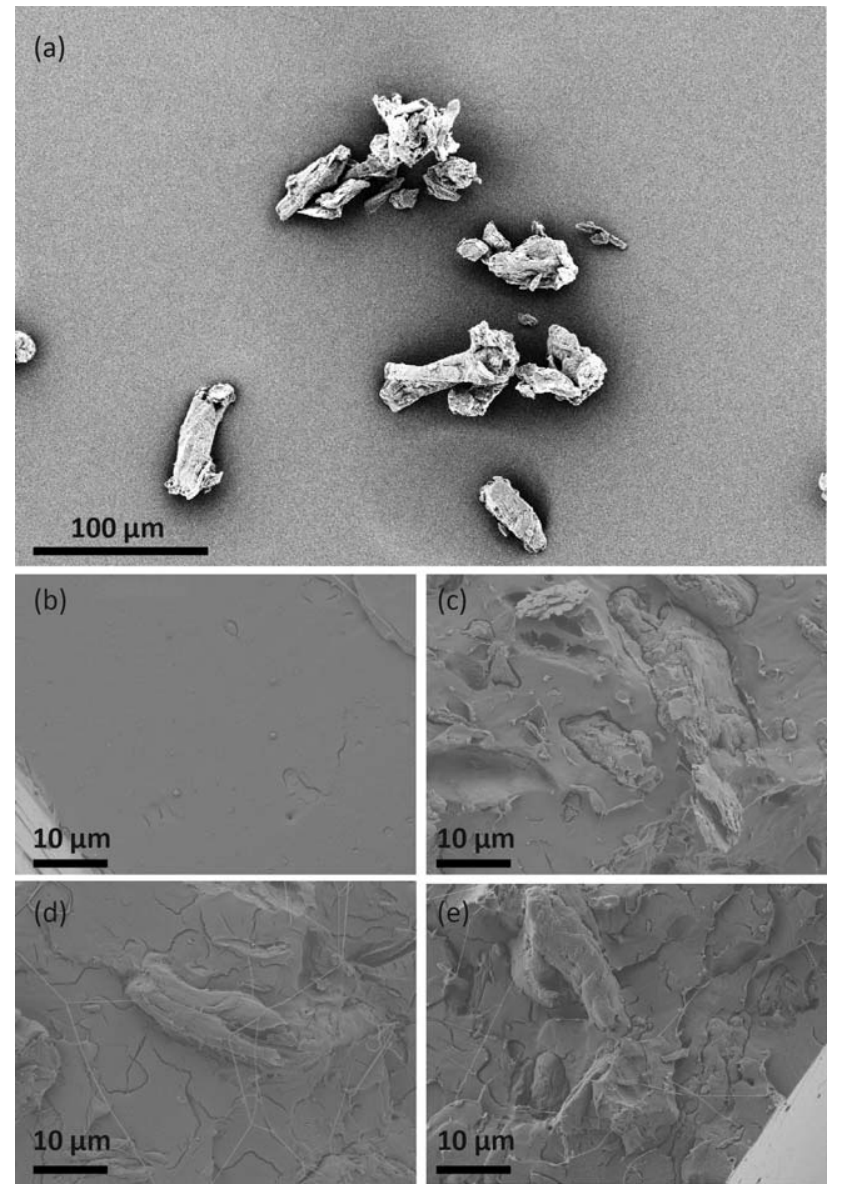

Figure 1. Scanning electron microscopy (SEM) images showing (a) sieved MCC particles sized 32-45 $\mu \mathrm{m}$; (b) cross-section of the pure PLA film; (c) cross-section of 80:20 PLA+MCC film; (d) cross-section of PLA+MCC film with XG $4 \mathrm{mg} \mathrm{g}^{-1}$ MCC; and (e) cross-section of PLA+MCC film with XG 50 $\mathrm{mg} \mathrm{g}^{-1}$ MCC. The scale bar is $100 \mu \mathrm{m}$ in the first image (a) and $10 \mu \mathrm{m}$ in the following images (b-e).

of MCC and had similar crystallinity. No impact on the total crystallinity of the final composite materials was observed for the varying amounts of XG added to each composite film. Because the addition of only $25 \mathrm{mg}$ XG to PLA resulted in an increased crystallinity, this increased crystallinity could be due to increased adhesion between the matrix and the filler materials in the composite.

The viscoelastic properties of the films were evaluated by oscillation of small deformations in the tensile mode at varying $\mathrm{RH}$ (Table II). The testing was performed at $25^{\circ} \mathrm{C}$ and the $\mathrm{RH}$ was increased from 10 to $90 \%$ over a period of $16 \mathrm{~h}$. Table II shows the storage modulus at $50 \% \mathrm{RH}$, which is a common environmental value, and the standard deviations obtained after at least three measurements. Taking into account the average deviations, no differences in G' and $\tan \delta$ were observed for the PLA, $\mathrm{PLA}+\mathrm{MCC}$, or PLA+XG films. The value of $2.7 \mathrm{GPa}$ in the pure PLA film is similar to the modulus $(2.8 \mathrm{Gpa})$ obtained for the material with the same composition of $\mathrm{PLA}+\mathrm{MCC}+\mathrm{XG}$, but in which the MCC did not go through the pre-absorption step. However, a continuous increase in the modulus was observed when MCC had been pre-covered with up to XG $25 \mathrm{mg} \mathrm{g}^{-1} \mathrm{MCC}$, showing a storage modulus of $2.7 \mathrm{GPa}$ for the pure PLA film compared with $3.3 \mathrm{GPa}$ for the XG $25 \mathrm{mg} \mathrm{g}^{-1}$ MCC film. Thus, the increase observed in moduli is only obtained for the materials where XG has been preadsorbed to the cellulose surface. Above XG 25mg g $\mathrm{g}^{-1}$ MCC, no further increase in mechanical properties is observed. Indeed, it is likely that the MCC surface is fully covered with XG at somewhere between 25 and $50 \mathrm{mg} \mathrm{XG} \mathrm{g}{ }^{-1}$ MCC, explaining why no or little difference in mechanical properties is expected between these films. This is in line with the higher adhesion between the materials obtained with XG. Values of $\tan \delta$ ranged between 0.016 and 0.024 for the materials at $50 \%$ RH. These values show that all materials are predominantly solid.

Viscoelastic properties as a function of $\mathrm{RH}$ are shown in Figure 2(a,b). Figure 2(a) shows the storage modulus upon increasing $\mathrm{RH}$ for the pure PLA, PLA+XG, and PLA+MCC with $25 \mathrm{mg}$ uncovered $\mathrm{XG}$. There is a slight decrease of the storage modulus for the PLA+XG film compared with the pure PLA film. The addition of MCC and non-adsorbed XG increases the storage modulus to some extent. It should, however, be noted that the differences between the materials are small when the standard deviation is taken into account. Figure 2(b) shows the storage modulus for the composite films with PLA+MCC and

Table I. Thermal Characteristics of PLA and the Composite Films in which PLA+XG was Dry Mixed Prior to Extrusion, PLA+MCC Contains PLA and MCC Particles with Preabsorbed XG (Ranging from 0 to $50 \mathrm{mg} \mathrm{g}^{-1} \mathrm{MCC}$ ), as Well as PLA+MCC+XG Uncovered, in Which the Preabsorption Step was Omitted

\begin{tabular}{lllll}
\hline Sample & $\operatorname{Tg}\left({ }^{\circ} \mathrm{C}\right)$ & Tc $\left({ }^{\circ} \mathrm{C}\right)$ & Tm $\left({ }^{\circ} \mathrm{C}\right)$ & Crystallinity $(\%)$ \\
\hline PLA & $51 \pm 2$ & $120 \pm 2$ & $147 \pm 0$ & $1 \pm 1$ \\
PLA+XG & $52 \pm 0$ & - & $147 \pm 1$ & $4 \pm 0$ \\
PLA+MCC & $50 \pm 1$ & $98 \pm 4$ & $148 \pm 0$ & $8 \pm 3$ \\
PLA+MCC+XG 4 & $51 \pm 1$ & $98 \pm 0$ & $148 \pm 1$ & $10 \pm 0$ \\
PLA+MCC+XG 10 & $50 \pm 1$ & $99 \pm 0$ & $148 \pm 0$ & $10 \pm 0$ \\
PLA+MCC+XG 15 & $51 \pm 2$ & $102 \pm 2$ & $146 \pm 4$ & $10 \pm 1$ \\
PLA+MCC+XG 25 & $51 \pm 2$ & $104 \pm 1$ & $145 \pm 3$ & $9 \pm 2$ \\
PLA+MCC+XG 25 uncov. & $52 \pm 1$ & $120 \pm 3$ & $146 \pm 0$ & $11 \pm 2$ \\
PLA+MCC+XG 50 & $50 \pm 1$ & $101 \pm 1$ & $147 \pm 1$ & $10 \pm 0$ \\
\hline
\end{tabular}


Table II. Mechanical Properties of PLA and the Composite Films in Which PLA +XG are Dry Mixed Prior to Extrusion, PLA + MCC +XG Contains PLA and MCC Particles with Preabsorbed XG (Ranging from 0 to $50 \mathrm{mg} \mathrm{g}^{-1} \mathrm{MCC}$ ), and PLA+MCC+XG Uncovered, in Which the Preabsorption Step was Omitted. All Measurements were Done at $T=25^{\circ} \mathrm{C}$

\begin{tabular}{lll}
\hline & $\begin{array}{l}\text { Tan } \delta \text { at } \\
\text { Storage modulus } \\
\text { at } 50 \% \mathrm{RH}(\mathrm{GPa})\end{array}$ & $\begin{array}{l}50 \% \mathrm{RH} \\
\left(\times 10^{-2}\right)\end{array}$ \\
\hline PLA & $2.7 \pm 0.1$ & $1.6 \pm 0.4$ \\
\hline PLA+XG & $2.6 \pm 0.1$ & $1.8 \pm 0.4$ \\
PLA+MCC & $2.8 \pm 0.2$ & $2.3 \pm 0.3$ \\
PLA+MCC+XG 4 & $3.0 \pm 0.1$ & $1.8 \pm 0.3$ \\
PLA+MCC+XG 10 & $3.0 \pm 0.1$ & $1.9 \pm 0.4$ \\
PLA+MCC+XG 15 & $3.1 \pm 0.1$ & $2.4 \pm 0.3$ \\
PLA+MCC+XG 25 & $3.3 \pm 0.0$ & $1.8 \pm 0.1$ \\
PLA+MCC+XG 25 uncov. & $2.8 \pm 0.2$ & $1.7 \pm 0.4$ \\
PLA+MCC+XG 50 & $3.1 \pm 0.2$ & $1.9 \pm 0.3$ \\
\hline
\end{tabular}

increasing amounts of pre-absorbed XG per gram of MCC. As can be seen in the figure, the increasing amount of $\mathrm{XG}$ increases the storage modulus up to XG $25 \mathrm{mg} \mathrm{g}^{-1} \mathrm{MCC}$. The storage modulus values are more or less constant for the different films up to $40 \%$ to $50 \% \mathrm{RH}$, demonstrating that the materials do not change at lower RHs. The storage moduli for $25 \mathrm{mg} \mathrm{g}^{-1}$ MCC covered and uncovered are different, hence the interface between MCC and PLA plays a role influence the visco-elastic properties of the films and could indicate an improved adhesion force between the filler and matrix, in agreement with the study of Raj et al. ${ }^{3}$ This improved adhesion force is thought to be related to capillary forces between the
XG and PLA at lower humidities, i.e. lower than $70 \% .^{3,33,34}$ It has further been proposed that changes in the topology of the cellulose filler can improve adhesion by mechanical anchoring of the PLA to the cellulose filler. ${ }^{13}$

The slopes of the storage modulus at an RH between 50 and $90 \%$ increased with more XG loading (Table III). These results show reductions in the strength of the material when the RH is higher than $50 \%$, which is most certainly explained by the presence of $\mathrm{XG}$ in the films and hence the reduction of capillary forces at higher $\mathrm{RH} .{ }^{3,33,34}$ A probable explanation is that the water accumulates in the more hydrophilic XG domain. Humidity cycles were performed on a selected film (15 mg XG/g MCC) to test whether any irreversible changes occurred in the film upon being subjected to high $\mathrm{RH}$ for a specific length of time (in total $16 \mathrm{~h}$ ). No hysteresis or change between the storage or loss moduli was observed between the different cycles of increasing and decreasing $\mathrm{RH}$ cycles (up to 3), indicating that no irreversible changes occurred in the material during the $\mathrm{RH}$ cycles performed within this study.

\section{Permeability Measurements}

To investigate their water permeability, films were placed between a donor and an acceptor chamber and the transport of tritiated water was measured over time. The mass transfer rate was calculated by plotting the accumulation of tritium-labeled water against time, as shown in Figure 3(a). This figure shows that the accumulation of water in the acceptor chamber is faster for films with higher amounts of added XG than for a film consisting of pure PLA. Permeability was calculated from the linear area in this plot according to eq. (3) and is presented in Figure 3 (b). The permeability for pure PLA has a value of $2.5 \times 10^{-13}$ $\mathrm{m}^{2} \mathrm{~s}^{-1}$, and when MCC was added the permeability increased to $6.0 \times 10^{-13} \mathrm{~m}^{2} \mathrm{~s}^{-1}$, which may be explained by the poor (a)

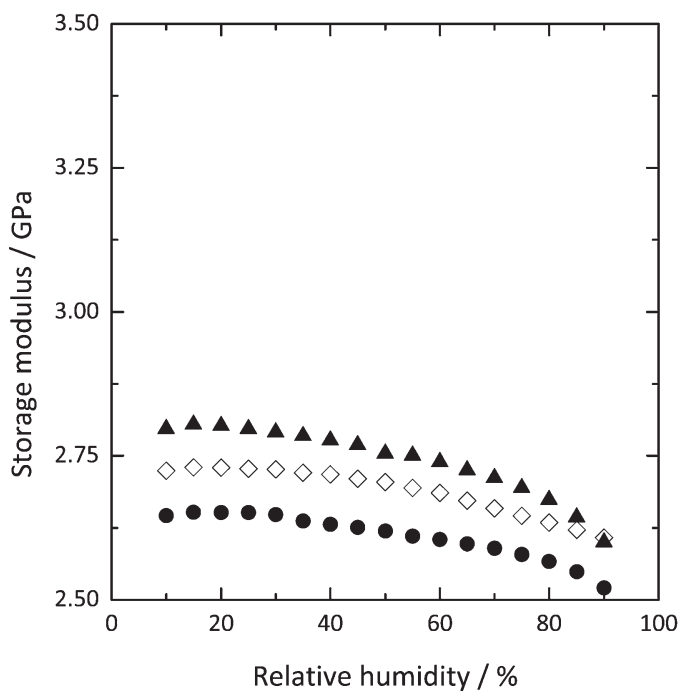

(b)

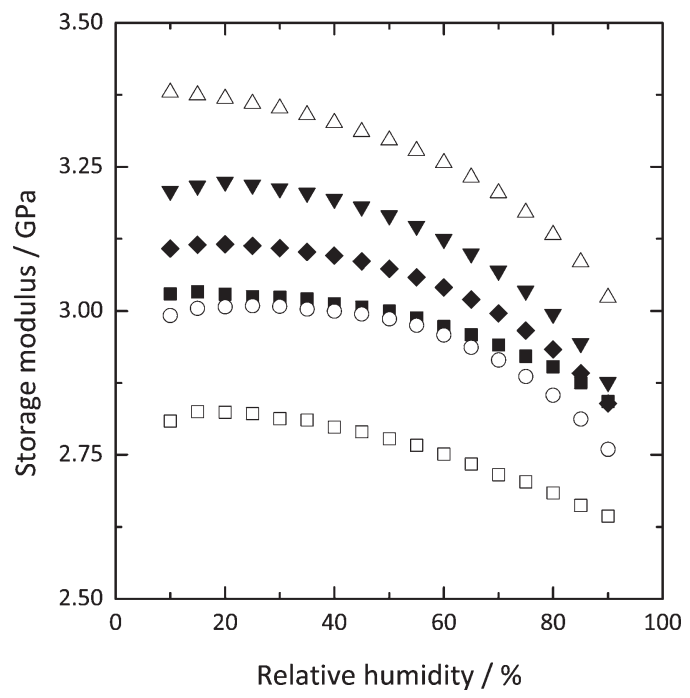

Figure 2. Storage modulus as a function of RH for (a) pure PLA films $(\diamond)$, PLA+XG films $(\bullet)$, and PLA+MCC with 25 mg uncovered XG/g MCC

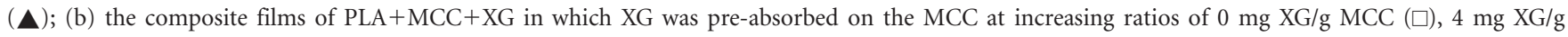
$\operatorname{MCC}(\boldsymbol{\square}), 10 \mathrm{mg} \mathrm{XG/g} \operatorname{MCC}(\bigcirc), 15 \mathrm{mg} \mathrm{XG/g} \mathrm{MCC}(\bullet), 25 \mathrm{mg} \mathrm{g}^{-1} \mathrm{MCC}(\Delta)$, and $50 \mathrm{mg} \mathrm{XG/g} \mathrm{MCC}(\boldsymbol{\nabla})$. Storage modulus of the films were obtained at $1 f=\mathrm{Hz}$, strain $=0.05 \%$, and $T=25^{\circ} \mathrm{C}$. 
Table III. The Effect of $\mathrm{RH}$ at $25^{\circ} \mathrm{C}$, Shown as the Slope of the Storage Modulus in the Range of $50-90 \%$ RH for the Pure PLA Film and the Composite Materials, Where PLA + XG was Dry Mixed Prior to Extrusion, PLA + MCC + XG Contains PLA and MCC Particles with Preabsorbed XG (Ranging from 0 to $50 \mathrm{mg} / \mathrm{g} \mathrm{MCC}$ ), as well as PLA+MCC+XG Uncovered, in Which Samples from the Pre-Absorption Step were Omitted. Measurements were Done Using $f=1 \mathrm{~Hz}$ and Strain $=0.05 \%$

\begin{tabular}{lll}
\hline Sample & Slope $\left(\times 10^{-3}\right)$ & $R^{2}$ \\
\hline PLA & -2.5 & 0.99 \\
PLA+XG & -2.2 & 0.94 \\
PLA+MCC+XG 0 & -3.4 & 0.99 \\
PLA+MCC+XG 4 & -3.8 & 0.98 \\
PLA+MCC+XG 10 & -5.5 & 0.96 \\
PLA+MCC+XG 15 & -5.7 & 0.96 \\
PLA+MCC+XG 25 & -6.7 & 0.96 \\
PLA+MCC+XG 25 uncov. & -3.7 & 0.93 \\
PLA+MCC+XG 50 & -7.0 & 0.96 \\
\hline
\end{tabular}

adhesion between cellulose and PLA reported elsewhere. ${ }^{3,8,10}$ For the films consisting of 4 or $10 \mathrm{mg} \mathrm{XG} \mathrm{g}^{-1} \mathrm{MCC}$, the permeability decreased or showed no difference to the PLA+MCC film. This can be explained by an improved adhesion between the matrix and the filler, which was suggested from the dynamic mechanical measurements. However, when higher amounts of $\mathrm{XG}$ are added, the permeability increases, which may be a result of the hydrophilic domains formed by the hemicellulose which covers the surface of the MCC. Reaching XG above 25mg XG $\mathrm{g}^{-1}$ MCC did not result in increased permeability, which is explained by the saturation limit expected to be reached at higher additions of $\mathrm{XG}^{31,32}$
These results should be compared with those for the film with $25 \mathrm{mg}$ of XG added to the PLA, which resulted in a water permeability of $5.8 \times 10^{-13} \mathrm{~m}^{2} \mathrm{~s}^{-1}$ (data not shown), more than twice as high as that of pure PLA. However, it should be remembered, as noted earlier, that an even dispersion of the XG in the PLA was difficult to achieve. This difficulty resulted in a large standard deviation compared to the other samples. The film that consisted of PLA, MCC, and $25 \mathrm{mg} \mathrm{XG}$, in which the MCC was not covered in advance showed a water permeability of $6.9 \times 10^{-13} \mathrm{~m}^{2} \mathrm{~s}^{-1}$. The $25 \mathrm{mg} \mathrm{XG/g} \mathrm{MCC} \mathrm{film} \mathrm{in} \mathrm{which}$ the MCC was covered in advance showed water permeability almost six times as high as pure PLA. The rise in water permeability is consistent with the explanation of the results obtained from the DMA analysis; water accumulates in the interface where the hemicellulose is sited. It should further be noted that crystallinity is high in the composite films, and the crystalline parts of a film are thought to decrease its permeability for smaller molecules such as water. This strengthens the theory that hydrophilic XG contributes to higher water permeability.

The permeability measurements indicate that water transport takes place along the surface of the MCC particles. The presence of XG domains between the PLA matrix and the MCC particles allows for increased water permeability at specific XG concentrations, as well as an increased storage modulus of the film. Our small deformation results support the observed increased adhesion between PLA + MCC + XG. The results further suggest that totally purified cellulose particles may not be the most suitable choice for the production of a renewable composite material, but that a mixture of PLA and cellulose obtained downstream in the cellulose extraction line is favorable for interfacial adhesion. A recent study performed by Marais and co-workers introduced the concept of grafting PLA onto XG, with the aim of improving the adhesion of MCC to the matrix (a)

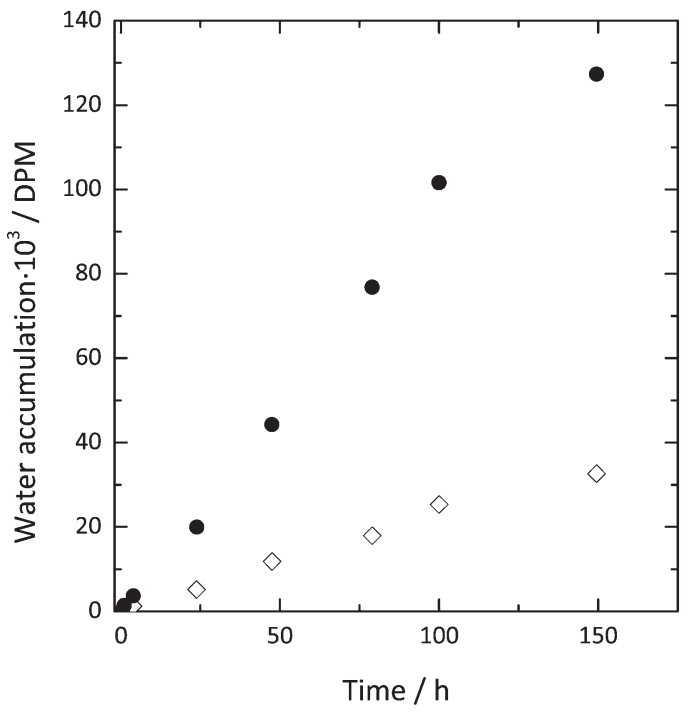

(b)

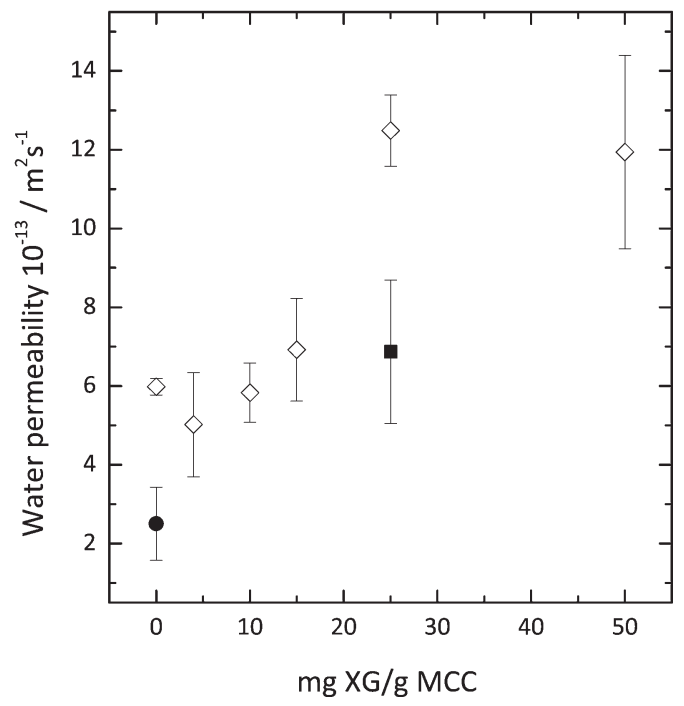

Figure 3. Data from permeability measurements showing (a) water accumulation as a function of time in the donor chamber for the pure PLA film ( $\diamond)$ compared with the composite film composed of PLA and MCC+XG particles (50 mg XG/g MCC) (O) and (b) water permeability of PLA and preabsorbed MCC + XG particles with increasing amounts of XG per gram MCC $(\diamond)$; the permeability of pure PLA $(\bullet)$ and composites of PLA + XG + MCC

) in which no pre-absorption step was done are added for comparison. 
of PLA $+\mathrm{XG}^{42}$ It would be interesting to compare the permeability of such materials with those obtained using the simpler approach adapted in this study, in which adsorption of XG on cellulose is promoted in order to improve the compatibility of PLA and MCC. Our permeability results suggest that such material would have increased permeability depending on the amount of XG required for improved mechanical properties.

\section{CONCLUSIONS}

In this study, renewable composite films of PLA, MCC, and XG were produced via extrusion and hot-melt pressing. It was shown that the addition of a hydrophilic hemicellulose increased the mechanical properties as well as the water permeability of the composite materials. However, these results were only observed when XG was pre-adsorbed to the surface of the cellulose prior to film production. Simply mixing the three components increased the films' water permeability, but no enhancement of their mechanical properties was observed. In addition, by covering the surface of MCC with XG via a simple adsorption step prior to blending with PLA, the dispersion of $\mathrm{MCC}+\mathrm{XG}$ in the PLA matrix was better than when the same amount of XG was non-adsorbed to the surface of MCC. Provided that the XG was pre-absorbed to the cellulose, water permeability of the final film was controlled by the content of XG added. Water transport is an important parameter to consider and control when developing new materials (e.g., those based on PLA and paper pulp), but also materials with distinct requirements for applications in biomedicine or agriculture. This study demonstrated how the water transport properties of PLA+MCC films can be varied by the site-specific addition of hydrophilic hemicellulose XG. These results may also be important for the production of totally biodegradable composite materials based on paper pulp, in which both cellulose and hemicelluloses can be present.

\section{ACKNOWLEDGMENTS}

Financial support from VINN Excellence SuMo Biomaterials (Supramolecular Biomaterials-Structure dynamics and properties) is gratefully acknowledged, as it the VINNMER grant to Anna Ström. The authors would further like to thank Michael Persson and Akzo Nobel for valuable discussions.

\section{REFERENCES}

1. Harding, K. G.; Dennis, J. S.; von Blottnitz, H.; Harrison, S. T. L. J. Biotechnol. 2007, 130, 57.

2. Vink, E. T. H.; Rábago, K. R.; Glassner, D. A.; Gruber, P. R. Polym. Degrad. Stab. 2002, 80, 403.

3. Raj, G.; Balnois, E.; Helias, M.-A.; Baley, C.; Grohens, Y. J. Mater. Sci. 2012, 47, 2175.

4. Kawamoto, H. Sci. Technol. Trends 2007, 22, 62.

5. Mathew, A. P.; Oksman, K.; Sain, M. J. Appl. Polym. Sci. 2006, 101, 300 .

6. Goffin, A.-L.; Raquez, J.-M.; Duquesne, E.; Siqueira, G.; Habibi, Y.; Dufresne, A.; Dubois, P. Biomacromolecules 2011, 12,2456 .
7. Jonoobi, M.; Harun, J.; Mathew, A. P.; Oksman, K. Compos. Sci. Technol. 2010, 70, 1742.

8. Mathew, A. P.; Oksman, K.; Sain, M. J. Appl. Polym. Sci. 2005, 97, 2014.

9. Oksman, K.; Skrifvars, M.; Selin, J.-F. Compos. Sci. Technol. 2003, 63, 1317.

10. Suchaiya, V.; Aht-Ong, D. Adv. Mater. Res. 2012, 488, 622.

11. Kale, G.; Auras, R.; Singh, S. P. J. Polym. Environ. 2006, 14, 317.

12. Bax, B.; Müssig, J. Compos. Sci. Technol. 2008, 68, 1601.

13. Baley, C.; Busnel, F.; Grohens, Y.; Sire, O. Compos. A Appl. S. 2006, 37, 1626.

14. Carlmark, A.; Larsson, E.; Malmström, E. Eur. Polym. J. 2012, 48, 1646.

15. Xiao, L.; Mai, Y.; He, F.; Yu, L.; Zhang, L.; Tang, H.; Yang, G. J. Mater. Chem. 2012, 22, 15732.

16. Raj, R. G.; Kokta, B. V.; Maldas, D.; Daneault, C. J. Appl. Polym. Sci. 1989, 37, 1089.

17. Wang, B.; Sain, M. BioResources 2007, 2, 371.

18. Altner, A. Available at: http://sodrapulplabs.com/. (Vol. 2013): Södra, (2013).

19. Armentano, I.; Dottori, M.; Fortunati, E.; Mattioli, S.; Kenny, J. M. Polym. Degrad. Stab. 2010, 95, 2126.

20. Douglas, G. H.; Sathiskumar, D.; Larry, C. W.; Karen, K. L.; Carol, M.; Debra, A. I. Degradable Polymers and Materials: Principles and Practice, 2nd ed.; American Chemical Society, Washington DC, 2012.

21. Moura, L. I. F.; Dias, A. M. A.; Carvalho, E.; de Sousa, H. C. Acta Biomater. 2013, 9, 7093.

22. Fortunati, E.; Peltzer, M.; Armentano, I.; Torre, L.; Jimenez, A.; Kenny, J. M. CORD Conf. Proc. 2012, 90, 948.

23. Pereda, M.; Amica, G.; Rácz, I.; Marcovich, N. J. Food Eng. 2011, 103, 76.

24. Sanchez-Garcia, M. D.; Lagaron, J. M. Cellulose 2010, 17, 987.

25. Sanchez-Garcia, M. D.; Gimenez, E.; Lagaron, J. M. Carbohydr. Polym. 2008, 71, 235.

26. Lagaron, J. M.; Catalá, R.; Gavara, R. Mater. Sci. Tech. 2004, 20, 1.

27. Suryanegara, L.; Nakagaito, A. N.; Yano, H. Compos. Sci.Technol. 2009, 69, 1187.

28. Mabasa Bergström, E.; Salmén, L.; Kochumalayil, J.; Berglund, L. Carbohydr. Polym. 2012, 87, 2532.

29. Zykwinska, A.; Thibault, J.-F.; Ralet, M.-C. Carbohydr. Polym. 2008, 74, 23.

30. Ahola, S.; Myllytie, P.; Österberg, M.; Teerinen, T.; Laine, J. BioResources 2008, 3, 1315.

31. Hayashi, T.; Marsden, M. P. F.; Delmer, D. P. Plant Physiol. 1987, 83, 384.

32. Zykwinska, A. W.; Ralet, M.-C. J.; Garnier, C. D.; Thibault, J.-F. J. Plant Physiol. 2005, 139, 397.

33. Raj, G.; Balnois, E.; Baley, C.; Grohens, Y . Colloids Surf. A 2009, 352, 47. 
34. Xiao, X.; Qian, L. Langmuir 2000, 16, 8153.

35. Drumright, R. E.; Gruber, P. R.; Henton, D. E. Adv. Mater. 2000, 12, 1841.

36. Henton, D. E.; Gruber, P.; Lunt, J.; Randall, J. In Natural Fibers, Biopolymers and Biocomposites; Mohanty, A. K., Misra, M.; Drzal, L. T., Eds. CRC Press: Boca Raton, 2005, 527.

37. Fortunati, E.; Armentano, I.; Zhou, Q.; Iannoni, A.; Saino, E.; Visai, L.; Berglund, L. A.; Kenny, J. M. Carbohydr. Polym. 2012, 87, 1596.
38. Hjärtstam, J.; Hjertberg, T. J. Appl. Polym. Sci. 1999, 74, 2056.

39. Larsson, M.; Hjärtstam, J.; Berndtsson, J.; Stading, M.; Larsson, A. Eur. J. Pharm. Biopharm. 2010, 76, 428.

40. Dickinson, E. Trends Food Sci. Tech. 1998, 9, 347.

41. Syrbe, A.; Bauer, W. J.; Klostermeyer, H. Int. Dairy J. 1998, 8, 179.

42. Marais, A.; Kochumalayil, J. J.; Nilsson, C.; Fogelstroem, L.; Gamstedt, E. K. Carbohydr. Polym. 2012, 89, 1038. 